REVISTA ANDALUZA DE ANTROPOLOGÍA.

NÚMERO 11: TRABAJO Y CULTURAS DEL TRABAJO EN LA GLOBALIDAD HEGEMÓNICA

SEPTIEMBRE DE 2016

ISSN 174-6796

[pp. 67-91]

http://dx.doi.org/10.12795/RAA.2016.11.04

Recibido: $17 / 5 / 16$

Aceptado: $12 / 6 / 16$

\title{
HOMBRES EN TRABAJOS REMUNERADOS DE CUIDADO: GÉNERO, IDENTIDAD LABORAL Y CULTURA DEL TRABAJO ${ }^{1}$
}

\section{YOLANDA BODOQUE}

Universidad Rovira i Virgili

MIREIA ROCA

Universidad Autónoma de Barcelona

DOLORS COMAS-D'ARGEMIR

Universidad Rovira i Virgili

\section{Resumen.}

En el presente artículo presentamos los primeros resultados de una investigación en curso sobre la participación de los hombres en el cuidado de personas adultas en situación de dependencia. En concreto nos centraremos en analizar las experiencias y percepciones de los hombres que tienen un trabajo remunerado de cuidados en organizaciones que operan en distintos sectores de la actividad económica, como servicios sociales, sanidad, servicio doméstico e, incluso, hostelería. Consideramos que dado el colapso existente en la atención a los cuidados de larga duración, que forma parte de lo que se ha calificado como crisis de los cuidados, deberá producirse una mayor implicación de los hombres, tanto en el ámbito familiar como en el laboral que es el que aquí nos ocupa. Conocer en qué circunstancias acceden a estos trabajos, cómo experimentan su actividad en relación

1. Esta investigación está financiada por RecerCaixa, un programa impulsado por la Obra Social 'la Caixa’ con la colaboración de la Asociación Catalana de Universidades Públicas (2014ACUP00045). 
al género o qué barreras culturales y de oportunidad limitan o favorecen la incorporación de los hombres en los trabajos remunerados de cuidado puede ayudar a favorecer esta implicación.

\section{Palabras Claves.}

Hombres cuidadores, cuidados de larga duración, cuidado social, género, trabajos de cuidado.

\section{Abstract.}

In the present article we present the initial results of an ongoing investigation into male participation in the care of adults in situations of dependency. Specifically, we analyse the experiences and perceptions of men professionally employed as paid care workers in organisations that operate in various sectors of the economy, including social services, health, home care and, even, the hotel sector. We believe that given the current collapse in long-term care provision, which forms part of the so-called 'care crisis', there should be greater involvement of men both in the family and professional setting and this is therefore the focus of the present study. Understanding the circumstances by which men enter these jobs, how they experience their work in relation to their gender and the cultural and employment barriers that limit or enable men to enter into paid care work will help strengthen their involvement.

\section{Keywords.}

Male care workers, long-term care, gender, care jobs.

\section{INTRODUCCIÓN}

El objetivo de este artículo es analizar la incorporación de hombres a los trabajos remunerados de cuidados. El incremento de personas con enfermedades crónicas o con discapacidades, así como el envejecimiento del envejecimiento han generado nuevas y crecientes necesidades de cuidados que no pueden ser atendidas únicamente desde el marco familiar y la implicación de las mujeres. Los cuidados de larga duración han pasado a tener una dimensión social y política. En España se aborda este problema más tarde que en otros países europeos: en el año 2006 se aprueba la Ley de Dependencia ${ }^{2}$, que el gobierno del Partido Popular modificada sustancialmente en el 2012 y hace fuertes recortes en su aplicación. Como consecuencia se produce una refamiliarización de los cuidados que va en paralelo a una creciente mercantilización de estos servicios, tanto

2. Ley 39/2006, de 14 de diciembre, de Promoción de la Autonomía Personal y Atención a las personas en situación de dependencia, más conocida como Ley de Dependencia. 
porque el retraimiento del estado facilita la expansión del mercado, como por la política de privatización de los servicios públicos (Deusdad et al., 2016).

El colapso existente en la atención a los cuidados de larga duración, que forma parte de lo que se ha calificado como crisis de los cuidados, obliga a replantearse la distribución desigual e injusta de este tipo de cuidado, que recae principalmente en las mujeres y está a cargo de la familia y solo subsidiariamente del Estado, el mercado o la comunidad. Deberá producirse una mayor implicación de los hombres en los trabajos de cuidados, tanto en el ámbito familiar como en el laboral que es el que aquí nos ocupa.

Los empleos de cuidados suponen una externalización del cuidado familiar y se caracterizan por su escaso valor social, precariedad laboral, feminización y etnización. Es en este contexto en el que nos proponemos analizar la presencia de hombres en trabajos remunerados de cuidados. Intentamos responder diversas preguntas relacionadas con las condiciones de acceso a los empleos de cuidados, las implicaciones para la identidad y las barreras que obstaculizan la incorporación de los hombres en este tipo de trabajos.

El concepto de culturas del trabajo desarrollado por Palenzuela (1995) y Moreno (1997) resulta útil en cuanto concilia las relaciones de producción, las relaciones de sexo-género y las relaciones interétnicas en la configuración de una matriz estructural identitaria que nos permite comprender prácticas sociales y cosmovisiones individuales y colectivas. Sin embargo, cuando tratamos los trabajos de cuidados, el concepto de culturas del trabajo queda desbordado por dos razones: en primer lugar, por el propio concepto de trabajo, puesto que las fronteras entre el trabajo no remunerado (familiar) y el remunerado (laboral) quedan muy desdibujadas e incluso contestadas, y en segundo lugar, porque se hace necesario incorporar los procesos de redefinición de una determinada cultura del trabajo en contextos cambiantes. Nuestra incursión en los trabajos de cuidados permite abordar esta complejidad.

La literatura académica feminista ha permitido dar visibilidad y asignar valor al tiempo de trabajo dedicado a las actividades no remuneradas que se ejercen en la familia y que contribuyen al bienestar colectivo (Carrasco, Borderías y Torns, 2011). Desde esta perspectiva se contesta la vinculación exclusiva del trabajo con las relaciones de producción, que invisibiliza los trabajos no remunerados que se realizan en el marco de relaciones de reproducción, y jerarquiza ambas dimensiones (Comas d’Argemir, 1995). Y es justamente esta vinculación de los cuidados con la familia y con las mujeres, lo que se proyecta en el ámbito laboral. La naturalización de los cuidados incide en la percepción de que no es necesaria una formación específica para realizarlos, resta valor a los empleos de este sector ocupacional y obstaculiza construir el conocimiento experto en este ámbito, de manera que la profesionalización de los cuidados choca con obstáculos importantes, quedando relegados a una mera ocupación laboral de baja cualificación, bajos salarios y escasas posibilidades de movilidad ascendente (Recio et al., 2015: 182). No solo el género 
explica esta escasa calidad de los empleos: también el origen de clase influye en que las mujeres los acepten y los vivan de forma satisfactoria. Y lo mismo podemos decir del origen étnico asociado a la condición de extranjería, de manera que los empleos de cuidados más precarios e intensivos (los realizados en los domicilios particulares) recaen mayoritariamente en personas inmigradas. Además, cuando el trabajo de cuidados se realiza en los hogares, la frontera entre lo familiar y lo laboral es todavía más difusa, puesto que los dos ámbitos se funden.

Respecto a las dinámicas procesuales: ¿qué sucede con la cultura del trabajo de cuidados cuando son hombres quienes se incorporan a este sector de empleos? Ha sido ampliamente constatado que, a diferencia de las mujeres que se introducen en ocupaciones masculinas, los hombres que trabajan en ocupaciones femeninas experimentan favoritismo y más fácil promoción. Mientras que la metáfora del "techo de cristal" sirvió para describir los obstáculos que viven las mujeres en el contexto laboral, la metáfora de la "escalera de cristal" ha reflejado las ventajas ocultas que experimentan los hombres en ocupaciones femeninas, tales como la preferencia en el acceso y la promoción, su fácil aceptación y la valoración positiva de sus aportaciones (Williams, 1992). Se constató que los hombres blancos son los principales beneficiarios de la "escalera de cristal", por lo que los estudios sobre segregación ocupacional no solo han de tener en cuenta el género, sino también la clase social, minorías étnicas o raciales y situaciones de discapacidad (Williams, 2013, 2015; Woodhams et al., 2015). Una perspectiva sintetizadora la aporta Smith (2012: 151) señalando que el "techo de cristal" y las "escaleras de cristal" se encuentran combinadas y, refiriéndose al contexto laboral y social de los Estados Unidos, afirma que raza, etnia y género se interseccionan para conformar un único mercado de trabajo con ventajas para los hombres blancos y desventajas para las mujeres y para las minorías.

La organización del trabajo en el contexto neoliberal aporta nuevas dimensiones, ya que instituye un individualismo competitivo que rompe con los modelos de organización productiva basados en la estabilidad del trabajador y el papel de los sindicatos en la consecución de derechos laborales. Por esto es importante situar las culturas del trabajo en el contexto de los cambios en el sistema capitalista y sus concreciones locales. Analizar los privilegios masculinos en los lugares de trabajo sin tener en cuenta la lógica de la explotación capitalista, hace perder la perspectiva analítica que permite reconocer cómo y porqué se producen desigualdades en los contextos laborales. Tal como señala Nancy Fraser (2011) las críticas a las desigualdades de género han de ir acompañadas de las críticas al capitalismo.

En este contexto de lógica neoliberal ¿continua funcionando la "escalera de cristal" para los hombres que se introducen en ocupaciones de mujeres? Nuestra hipótesis es que en el caso de los trabajos de cuidados sí funciona, pero de forma mucho más débil respecto a lo que se ha mostrado en profesiones como la enfermería, el magisterio o el trabajo 
social. Se trata todas ellas de profesiones feminizadas, pero los trabajos de cuidados, se configuran como empleos más tarde, tienen mayores dificultades para profesionalizarse en la medida en que carecen de cualificación y la posibilidad de carrera profesional es muy limitada. Trataremos de mostrar cómo los condicionantes con los que se han configurado los empleos de cuidados limitan drásticamente el alcance de la "escalera de cristal".

Otra de las dimensiones que hay que considerar en la introducción de los hombres en los empleos de cuidados son las implicaciones para la identidad, en la medida en que se confrontan con los modelos de masculinidad hegemónica (Connell y Messerschmidt, 2005), entendiendo estos también en su diversidad, cambios y perspectiva interseccional. Podemos señalar que una vertiente de la masculinidad hegemónica se ha construido como care-free (Hanlon, 2012), pues el distanciamiento de los hombres respecto al cuidado hace que puedan dedicarse al trabajo remunerado o actividades de prestigio. Nos preguntamos pues cómo los hombres que están empleados en el sector de cuidados negocian su concepción de la masculinidad en el lugar de trabajo.

Nuestra reflexión en este artículo aporta elementos para la comprensión de los procesos de segregación y discriminación ocupacional que continúan teniendo fuerte presencia en nuestra sociedad. Hay que señalar que desde la década de los años 80 las mujeres han incrementado de forma notable su presencia en el mercado de trabajo y, además, las mujeres de clase media se han introducido en profesiones tradicionalmente masculinas que previamente les habían estado vetadas, alcanzando incluso la mayoría en algunas de ellas (medicina, magistratura, farmacia). No sucede así a la inversa, pues los hombres se han introducido muy poco en ocupaciones feminizadas y Williams (2013) constata que las cuatro profesiones que estudió en 1992 (enfermería, biblioteconomía, magisterio y trabajo social) continúan presentando una fuerte segregación de género veinte años después.

$\mathrm{Al}$ analizar la introducción de los hombres en los trabajos de cuidados tomamos en consideraciónlasdesigualdadesenloslugaresdetrabajoylasnocionesdeinterseccionalidad y de masculinidad hegemónica. Esto permite abordar las construcciones culturales que diferencian hombres y mujeres en sus experiencias laborales y las que modelan la identidad masculina en los pocos hombres que cruzan las fronteras de género y realizan trabajos en que predominan las mujeres (West y Zimmerman, 1987). Todo ello en el contexto de cambios en el sistema capitalista orientados a la lógica neoliberal. Constataremos que muchos hombres llegan a estas ocupaciones como fruto de la grave crisis de empleo, de manera que han tenido que aceptar empleos que no se habían planteado realizar.

A partir de estas consideraciones los objetivos específicos de este artículo se centran en las siguientes dimensiones: 1) Motivaciones de los hombres para acceder y mantenerse en empleos de cuidados, y 2) Experiencias de los hombres en los trabajos de cuidados 
en su calidad de grupo minoritario. Para el análisis tendremos en cuenta las barreras culturales (construcciones de género) y de oportunidad (posición en el mercado de trabajo) que limitan la incorporación de hombres a los trabajos de cuidados y los factores que contribuyen a romper estas barreras.

Nuestra investigación se ha centrado en Cataluña. Hemos realizado entrevistas a hombres cuidadores y observaciones en el lugar de trabajo como estrategias metodológicas básicas. Para redactar este artículo nos hemos basado en once entrevistas en profundidad realizadas a hombres entre 25 y 58 años, siendo mayoritaria la franja entre 45 y 58 años. 8 de ellos son españoles y 3 latinoamericanos. Solo uno de ellos tiene estudios universitarios, pues predominan formaciones básicas como el graduado escolar, formación profesional de grado medio y formación profesional ocupacional. Llevan trabajando como cuidadores entre dos y siete años, pues la mayoría accede a esta clase de trabajo en el contexto de la crisis económica, sustituyendo empleos anteriores. Trabajan en centros residenciales, centros de día o en servicios de atención domiciliaria (SAD) y algunos combinan su trabajo en una empresa con trabajos en domicilios particulares. Aunque no utilizamos aquí la muestra completa de hombres entrevistados en nuestra investigación, consideramos que es suficientemente significativa y supone un avance de los primeros resultados.

\section{ANTECEDENTES Y ESTADO DE LA CUESTIÓN}

La literatura académica sobre los trabajos de cuidados y su profesionalización muestra que el foco atención se ha dirigido fundamentalmente hacia las mujeres ya que efectivamente son ellas quienes ocupan buena parte de este sector laboral. Algunos autores muestran cómo ha sido principalmente el incremento de las necesidades de cuidados de larga duración lo que ha dado lugar a un creciente proceso de institucionalización y profesionalización de los trabajos de cuidados sin que ello haya implicado la desaparición del cuidado familiar (Lethbridge, 2013; Rogero-García y Ahmed-Mohamed, 2014; Pickard, 2012). El trabajo de cuidados se lleva realizando desde distintos sectores de la actividad económica, como servicios sociales, sanidad, servicio doméstico e, incluso, hostelería. Sin embargo, las evidencias empíricas muestran que los rasgos del trabajo familiar se proyectan en los empleos de cuidados, lo cual parece explicar una presencia mayoritaria de mujeres, condiciones laborales precarias, escasa visibilidad y escasa valoración social (Torns et al., 2014).

Hay que destacar la ausencia de estudios sobre la presencia de hombres en los trabajos remunerados de cuidados, lo que contrasta con otras ocupaciones también feminizadas. En todo caso, es interesante tener en cuenta los hallazgos realizados en los estudios que han tratado la enfermería, el magisterio y el trabajo social, algunos de los cuales se han centrado en comprender las formas en que los hombres experimentan, comprenden y afrontan los desafíos de su masculinidad y en analizar las dinámicas de poder basadas en 
el género (Shen-Miller y Smiler, 2015). Son interesantes aquellos focalizados en analizar las estrategias para reclutar y retener a los hombres en estas profesiones de manera que dejen de pensarse fundamentalmente como profesiones de mujeres. Es el ejemplo del estudio que recientemente llevó a cabo Cottingham (2014) con enfermeros. Esta autora considera que a pesar de los cambios en las dinámicas de género los hombres continúan siendo minoritarios en las profesiones del cuidado relacionado con la salud. Esta presencia minoritaria puede ser debida a que en la profesión se requiere una serie de habilidades (empatía, compromiso emocional y ayuda a los demás) que son la antítesis de las nociones hegemónicas de ser hombre (duro, emocionalmente distante, individualista, racional y técnicamente competente). A partir de esta premisa analiza las estrategias que utilizan las organizaciones para captar a los hombres en esta profesión: desde aquellas que resaltan los aspectos más hegemónicos de la masculinidad como fundamentales para contrarrestar el trabajo de sus compañeras, a aquellas que muestran cómo las masculinidades alternativas aportan elementos positivos al desarrollo de la enfermería. Quienes fueron cooptados por la vía de la masculinidad hegemónica resaltan lo difícil que les resulta conciliar la masculinidad convencional con el cuidado. Los cooptados por la vía de las masculinidades alternativas, en cambio, resaltan la importancia del cuidado, reivindicando que la crianza y la prestación de atención no son exclusivamente femeninas. De esta manera es como la autora demuestra que se continúan reproduciendo roles segregados entre hombres y mujeres porque resulta complicado conciliar la masculinidad hegemónica con las actividades asociadas a los cuidados (Cottingham, 2014). Ruth Simpson (2004) realizó un estudio de características similares aunque poniendo el acento en el itinerario laboral de los hombres antes de acabar siendo bibliotecarios, azafatos, enfermeros o maestros. Definió tres perfiles de hombres: los que buscan activamente dedicarse a esta profesión (a los que llamó solicitantes), los que la encuentran por casualidad y la consideran provisional (los buscadores) y, finalmente, los que llegan a la profesión después de haber trabajado en ocupaciones consideradas masculinas y deciden dedicarse a ella (los colonos). Todos ellos, dice Simpson (2004), se benefician de su condición minoritaria mediante la suposición de que mejorará su liderazgo (lo que denomina el efecto de la autoridad innata); a través del trato diferencial (el efecto de consideración especial); o porque actúan de manera arribista (el efecto carrera). En general, todos ellos se sienten cómodos trabajando con mujeres (el efecto comodidad) pero adoptan una serie de estrategias para restablecer cualquier indicio de debilitamiento de su masculinidad renombrando su categoría, distanciándose de las características asociadas a lo femenino e intentando mejorar su posición laboral en la organización. Christensen y Knight (2014) también muestran como, a pesar de las barreras emocionales, verbales o sexuales que los hombres encuentran cuando acceden a estas profesiones, en sus narrativas (trabajaron con estudiantes de enfermería) expresan que acceden a ellas porque, a pesar del marcador de género que poseen, les parece una 
ocupación flexible y prometedora que les permite desarrollar sus límites profesionales aprovechando las ventajas que les supone ser minoría, por ejemplo, y exteriorizando su heterosexualidad.

Una muestra más del desencuentro entre la masculinidad hegemónica y los cuidados nos la proporciona Evans (1997) que también demuestra que en la enfermería se da una alta proporcionalidad de hombres que se distancian de las actividades de cuidado directo para situarse en posiciones administrativas de élite por varias razones: por la necesidad de separar lo que es masculino de las actividades propias del cuidado (por ser consideradas femeninas); para distanciarse de sus compañeras y de la imagen femenina por excelencia de la enfermera; porque las propias instituciones y organizaciones reproducen imágenes que perpetúan la ventaja masculina; o, incluso, porque las propias compañeras favorecen (directa o indirectamente) que los hombres accedan a estas posiciones.

Aunque de forma minoritaria, los hombres también han entrado en el sector de los cuidados, de la misma manera que lo hicieron en ocupaciones tradicionalmente feminas, como el trabajo social, magisterio, biblioteconomía o enfermería. De hecho se trata de una presencia tan reducida que ni siquiera ha llamado la atención de quienes investigan sobre los trabajos remunerados de cuidado (que han centrado su atención en las mujeres trabajadoras). En cambio, sí se han realizado numerosos estudios sobre hombres que cuidan a sus familiares (véase entre otros Calasanti y Bowen, 2006; Ducharme et al, 2006; Milne y Hatzidimitriadou, 2003; Ribero, 2005), así como sobre hombres migrantes en trabajos de cuidado, que no se estudian por su condición de cuidadores sino por el hecho de ser migrantes con dificultades de inserción laboral (véase entre otros Catanzaro y Colombo, 2009; Haile y Siegman, 2014; Quartararo y Falcinelli, 2013; Scrinzi, 2005). Podemos pensar que la escasa atención prestada desde la academia a la presencia de hombres en los trabajos de cuidados puede ser debida a lo que afirmábamos al inicio de este apartado: que los rasgos del trabajo familiar y doméstico se proyectan sobre el trabajo remunerado de manera que su interés se ha dirigido principalmente a las mujeres por ser las que mayor presencia tienen así como en su falta de visibilización y valoración.

\section{TRABAJOS DE CUIDADOS EN ESPAÑA, POLÍTICAS SOCIALES Y ORGANIZACIÓN DE LOS SERVICIOS}

El despliegue de un nuevo modelo europeo de protección social basado en derechos de ciudadanía en la atención a la dependencia ha significado una nueva concepción social de la responsabilidad pública del cuidado: de un modelo de cuidado asistencial a un modelo universal. Varios cambios sociodemográficos (envejecimiento de la población y fuerte presencia de las mujeres en el mercado laboral), suponen un crecimiento de las necesidades de cuidado y una reestructuración de los Estados del Bienestar. En Europa se despliegan diferentes fórmulas para dar respuesta a las necesidades de cuidado: políticas de contención de costes, desplazamiento de riesgos sociales al mercado, a la familia y 
a los individuos, lo que supone una feminización del cuidado y el protagonismo del cuidado informal (Durán, 2014; Marbán, 2011; Rodríguez, 2011).

En España la atención a los cuidados se ha basado tradicionalmente en la centralidad de la familia, la subsidiariedad del Estado (escasa cobertura de servicios sociales públicos) y la residualidad del mercado (Comas d'Argemir, 2015; Martínez-Bujan, 2014; Rodríguez, 2007; Tobío et al., 2010). Con la creación de la Ley de Dependencia, aparece el derecho subjetivo a recibir cuidado a fin de garantizar la cobertura de estas necesidades a toda la población. En Catalunya el sistema público de servicios sociales está regulado, además, por la Ley de Servicios Sociales ${ }^{3}$. Son prestaciones de servicios, económicas y tecnológicas que conforman una red de servicios sociales de responsabilidad pública. Aunque los objetivos de las políticas europeas de protección social de la dependencia (universalización, suficiencia y calidad y sostenibilidad) están recogidos por la ley española, y a pesar de que la ley de Dependencia supuso un gran avance en relación a los derechos sociales, ya en un inicio hubo dificultades en su aplicación (Rodríguez, 2007). Se crearon grandes expectativas pero la entrada de la ley coincide con el inicio de la crisis económica, que dificulta la universalidad en la cobertura de los derechos a la vez que aumentan las desigualdades sociales (Deusdad et al., 2016; Peterson, 2015). Además, el aumento de las necesidades de cuidado (por el envejecimiento de la población), sumado a las políticas de los gobiernos conservadores, no ha favorecido la sostenibilidad del sistema. Desde 2010 se aplican diversas reformas legales que inciden negativamente en la cobertura del cuidado debilitando los derechos reconocidos en un inicio (Vilà, 2013) y en el 2011, bajo el gobierno socialista, comienzan las reformas estructurales para recortar el gasto social. Sin embargo, es el Partido Popular el que instaura políticas basadas en una lógica neoliberal que prescriben la austeridad y la rentabilidad de la política social, legitimando recortes en las prestaciones y los servicios de atención para las personas dependientes y cambiando el foco de las necesidades de atención al del déficit público (Peterson, 2015). Se reducen los servicios sociales y la prestación económica de asistencia se generaliza, lo que favorece a los proveedores privados y potencia el cuidado familiar, dando lugar a un retroceso en el despliegue público de servicios, aumentando las desigualdades sociales y las necesidades no cubiertas (Deusdad et al., 2016; Marbán, 2011; Martínez-Buján, 2014). La presencia del mercado va en aumento, ya sea por la compra del cuidado como para la gestión privatizada de los recursos públicos, que ha encontrado en este sector un nuevo lugar para la obtención de beneficios, estrechamente ligado a la feminización del trabajo, y a la precariedad laboral (Pérez, 2006). Las políticas de atención al cuidado de larga duración son pues un complemento al cuidado informal, refuerzan el mercado y no alteran la estructura de género (Comas de Argemir, 2015). En España, los principales agentes proveedores de cuidado son los cuidadores familiares

3. Llei $12 / 2007$, d'11 d'octubre, de Serveis Socials. 
no remunerados, los empleados por las Administraciones Públicas y los remunerados privadamente a través del mercado de servicios (Durán, 2014). La forma más común de atención es el cuidado familiar no remunerado (representa el 78,5\% del cuidado); un $10,9 \%$ de la población recibe únicamente cuidado remunerado (el 7,3\% privado); y el $10,5 \%$ recibe indistintamente cuidado remunerado y no remunerado (Rogero-García y Ahmed-Mohamed, 2014).

Con el despliegue de los servicios sociales de atención a la dependencia, se contempla que los servicios tengan capacidad para crear puestos de trabajo. Según el Libro Blanco de la dependencia (2004), ya se preveía que la generación bruta de empleo del sistema de atención a la dependencia superaría los 350.000 puestos de trabajo (260.000 netos) en la prestación directa de servicios. La ley se proponía construir un sistema público universal basado en el cuidado profesional, pero la realidad está siendo otra, entre otros factores porque no se han priorizado los servicios ante las prestaciones económicas. Las ramas de actividad por ámbito de actuación de la ley son: las actividades sanitarias, las de servicios sociales y los hogares que contratan personal doméstico. Los límites entre los tres niveles son difusos y se basan en una feminización del sector, una escasa valoración social y del mercado de trabajo, y unas condiciones laborales precarias (Otaegui, 2007, Recio, 2010; Recio et al., 2015). Los profesionales del cuidado tienen formaciones muy diversas, muchas de ellas no regladas. En Cataluña no es hasta 2003 que se crea la titulación de Técnico en Atención Sociosanitaria ${ }^{4}$ y se reconoce la actividad laboral dentro de la formación reglada. Corresponde a un ciclo formativo de Grado Medio de 2000 horas y unifica bajo un mismo nombre a todos aquellos trabajos que intervienen en la atención a la persona. Debido a la falta de trabajadores/as cualificados en el sector y a la creciente demanda de empleo, el 27 de noviembre de 2008 el Consejo Territorial aprueba el Acuerdo gubernamental ${ }^{5}$ que recomienda que las gerocultoras y auxiliares de ayuda a domicilio tengan una formación específica (Formación Profesional) o que puedan acreditar la experiencia laboral y la formación no reglada ${ }^{6}$ (proceso de reconocimiento de competencias). Se produce un importante aumento del empleo en los servicios residenciales y en los servicios de atención diurna (teleasistencia, servicios

4. Real Decreto 496/2003, de 2 de mayo, por el que se establece el título de Técnico en Atención Sociosanitaria y las correspondientes enseñanzas comunes (BOE núm. 124, 05/24/03). Posteriormente reformado por la ley catalana: LOE: Real Decreto 1593/2011, de 4 de noviembre, por el que se establece el título de Técnico/a en Atención a personas en situación de dependencia y se fijan sus enseñanzas mínimas.

5. Resolución 2 de diciembre de 2008, publicada en el BOE de 17 de diciembre.

6. Mediante el Real Decreto 1224/2009, de 17 de julio, de reconocimiento de las competencias profesionales adquiridas por la experiencia laboral (BOE, 25 de agosto 2009) para equiparar a los profesionales cualificados. Se requiere experiencia laboral (2.000 horas trabajadas, en los últimos 10 años) y/o formación en competencias profesionales (300 horas en los últimos 10 años), tener residencia española y más de 20 años. 
de ayuda a domicilio y centros de día). Así, en el año 2009 había en Catalunya 37.400 personas asalariadas en el sector: el 1,2\% de la población ocupada. Del total, $88 \%$ son mujeres, de un promedio de edad de 41 años, y el 54\% son auxiliares de gerontología. Aumenta sobre todo el empleo en el sector privado, que alcanza el 79,4\% de la ocupación (un $56,9 \%$ en entidades mercantiles y un $22,5 \%$ en entidades sociales), mientras que los empleos de cuidados en entidades públicas son considerablemente menos, un 20,6\% del total (Informe ACRA, 2013). Son trabajos poco cualificados y con dificultades para su profesionalización debido a básicamente a unos imaginarios colectivos que proyectan unas representaciones sociales familiares y femeninas (Recio et al., 2015), así como un sistema de servicios que requiere de actividades con poca cualificación (Fantova, 2013).

Las políticas públicas tienen un papel crucial en la construcción del trabajo de cuidados así como también la estructura del mercado laboral, ya que influyen en su valoración y en el estatus de trabajadoras y cuidadoras (Esquivel, 2011; Razavi y Staab, 2010; Recio, 2010; Peterson, 2015). Ante el incremento de la demanda de cuidadores profesionales y el déficit de trabajadores cualificados, de acuerdo con la tendencia a nivel Europeo, se prevé el refuerzo de las políticas de formación y una mayor regulación del marco legal y de las condiciones laborales de los cuidadores (Marbán, 2011).

\section{MOTIVACIONES DE LOS HOMBRES PARA ACCEDER Y MANTENERSE EN EMPLEOS DE CUIDADOS}

A pesar de la escasa presencia de hombres en los trabajos remunerados de cuidados no nos ha resultado difícil encontrar algunos dispuestos a explicarnos su experiencia como cuidadores en los distintos ámbitos donde ejercen sus actividades.

Preguntados por su itinerario laboral y personal hasta llegar a ejercer como cuidadores, la mayor parte nos relataron un periplo similar que se inicia con los graves problemas con los que muchos se encontraron en el inicio de la reciente crisis económica: una dilatada experiencia laboral en profesiones relacionadas con el transporte, la construcción o diversos sectores industriales, en empresas que en pocos años fueron cerrando, dejándoles en el paro y con escasas o nulas posibilidades de volver a trabajar en lo mismo. Tomemos como ejemplo el caso de Fernando. Tiene 50 años, está casado y tiene un hijo. Durante treinta años estuvo trabajando en el sector de la construcción haciendo diferentes trabajos, desde albañil a agente inmobiliario, muy bien pagados hasta que llegó la crisis. La empresa cerró, pero continuó durante algunos meses haciendo "remiendos, chapuzas" hasta que llegó un momento en que dejó de trabajar. Decide entonces reciclarse siempre dentro del sector que mejor conocía, por lo que hace un cursillo de gestor de almacén, pero no consiguió ningún empleo, hasta que un día dirigió su atención hacia un curso para trabajadores familiares: "entonces vi mi salvación y pregunté, esto qué es? y me dijeron que es como la geriatría, personas que cuidan personas y que este trabajo tiene muchas salidas (gesto de dinero)". 
Un itinerario laboral y personal similar al de Carles $^{7}$ con la única diferencia que se queda sin trabajo por decisión de la empresa, que decide hacer reajustes y contratar personal más joven. Carles decide voluntariamente cambiar de ámbito laboral y prepararse para ser celador de hospital. Por motivos que desconoce no es posible acceder a este curso y en la propia oficina de empleo le recomiendan realizar el curso de formación ocupacional de auxiliar de geriatría y, como Fernando, vuelve a hacerse la misma pregunta "¿Qué es eso?", obteniendo también la misma respuesta "es eso, ayudar a la gente mayor, trabajar en una residencia". Es decir, es cuando deciden reciclarse laboralmente que la mayor parte de nuestros informantes tienen su primer contacto con los empleos de cuidados.

Deciden probar suerte entre otras cosas porque no disponen de un abanico de posibilidades laborales: "...el paro se me estaba acabando y lo intenté como una salida más de la vida laboral” (Carles); "...nunca me hubiera imaginado en este sitio, jamás, yo jamás me hubiera imaginado. Ya te digo, pero vi aquello y bueno, estaba cobrando el paro en aquel entonces pero a mí se me acababa el paro en marzo de 2010" (Fernando). Por su parte Joan, según nos cuenta, se vio obligado a hacer el cursillo para poder cobrar la prestación de desempleo. Él habría elegido el de energías renovables o el de alemán, pero solamente pudo optar al de auxiliar de geriatría.

Nuestros informantes se forman como cuidadores utilizando vías diferentes. Pedro realiza un cursillo con el que obtiene un certificado de profesionalidad para atender personas dependientes. Cree que su experiencia como cuidador de su padre le ayudará aunque considera que para cuidar no se necesitan demasiados conocimientos. Idea que se confirma cuando comprueba que los compañeros/as del cursillo no tienen un buen nivel de formación básica. También Carles tenía experiencia en el cuidado de su madre y de su esposa y considera que esta experiencia más las habilidades que adquiere con las actividades cotidianas del cuidado doméstico, le fueron útiles en la formación que duró seis meses así como en el trabajo que consiguió inmediatamente después y en el que continúa en la actualidad.

Arturo forma parte de quienes que se ven obligados a cambiar de trabajo y se decantan por el de cuidados. No obstante decide realizar una formación más completa y cursar un módulo de formación profesional de grado medio con una duración de dos años, lo cual le permite opinar en contra del hecho de que no exista un buen y único plan de capacitación de las personas que se dedican a los trabajos de cuidados puesto que va en contra de la dignificación de la profesión.

Pablo y Jaume, en cambio, ya iniciaron su itinerario laboral atendiendo a su interés por las actividades de cuidado (entrarían dentro de la categoría de solicitantes, según la clasificación de Simpson, 2004). El más veterano, Jaume, es auxiliar de enfermería. De

7. Las entrevistas realizadas a Carles, Arturo, Pablo, Aldo, Robert, Pedro, Joan y Albert originalmente son en catalán. La traducción es nuestra. 
Jaume nos llamó la atención el hecho de que desde joven (acompañando a su madre) y hasta hoy (como sueldo complementario) realice cuidados paliativos a personas que deciden ir a morir a sus domicilios ("hacer velas es otra forma de cuidar") y que haya sido precisamente esta actividad la que le dirigió a su profesión actual. De manera similar, de Pablo no nos interesa tanto que está a punto de terminar el grado de enfermería, sino el hecho de que se esté pagando sus estudios cuidando a personas mayores en sus domicilios. Es importante remarcar que su trayectoria como cuidador también se inicia en casa, asumiendo el cuidado de su padre cuando el resto de sus cuatro hermanos no se vieron capaces de hacerlo:

"No sé si fue instintivo o no, pero fui uno de los que más carga soportó (...) creo que no lo decidí. Me salió solo. De hecho siempre había querido, no sé por qué, trabajar en un banco. Y por el tema notas no llegué a hacer la selectividad y la única vía que tenía de salida era hacer el grado este (...) A mí siempre me ha gustado mucho tratar a la gente, cuidar a la gente y ayudarlos (...) Y mientras estaba haciendo la carrera sucedió lo de mi padre y todavía potenció más las ganas de seguir en esta profesión".

No nos faltan ejemplos de hombres que realizando otro tipo de trabajos, sin ninguna relación con el cuidado, sufren una crisis personal de la que salen a flote cambiando radicalmente de trabajo. Son nuestros ejemplos de colonos, que se sienten reconfortados con su nueva actividad (Simpson, 2004). Aldo nos comenta que cuidar a otras personas le ayuda a "realizarse" de una manera más satisfactoria que trabajando en un laboratorio rodeado de microscopios: "me gusta... ayudar a las personas y estar en contacto con las personas. Y sentirme realizado de una manera diferente a como yo venia sintiéndome realizado hasta ahora". Quizás Robert no sufrió una crisis personal, pero verse realizando una actividad complementaria como es un voluntariado en Cruz Roja, le iluminó su itinerario laboral: "trabajando aquí me di cuenta que me gustaba mucho, me llenaba mucho poder estar con una persona, darle la ayuda que quizás 'en aquel momento' él no podía tener por sí mismo".

Tanto para quienes se dedican desde el inicio de su itinerario laboral como para quienes se han incorporado más tarde, el trabajo de cuidados ha resultado una buena oportunidad laboral. En su caso ha funcionado la "escalera de cristal", por la facilidad con que pudieron acceder al empleo. En las propias oficinas de empleo ya se les sugiere que su condición de hombres les facilitaría insertarse de nuevo en el mercado laboral: "La del paro me decía éste trabajo tiene muchas salidas... y para los hombres más, porque los hombres van más buscados en este tipo de trabajos"' (Fernando).Y, ciertamente, nuestros entrevistados no tardaron en empezar a trabajar e incluso disponer de un abanico de posibilidades donde poder elegir: residencias asistidas, servicios de ayuda a domicilio, sociosanitarios, domicilios particulares, etc. Joan acabó el curso en 2012 e inmediatamente empieza a trabajar en un SAD y en dos domicilios privados; Fernando acabó el cursillo en marzo 
de 2010 y en junio empezó a trabajar en la residencia (donde actualmente sigue); Carles, terminó en 2014 y empieza a trabajar donde realizó las prácticas y a ser reclamado en varios servicios: "Lo importante es tener trabajo y por eso cuando me llamaron para decirme que tenía que trabajar julio y agosto dije: yo lo que quiero es trabajar"; Arturo, que trabaja en la misma residencia asistida desde 2009, ni siquiera había terminado su periodo de formación cuando tuvo la oportunidad de incorporarse.

Quienes ya tenían experiencia como cuidadores en casa no les resultó difícil acomodarse en el trabajo: "como en casa lo he visto todo o casi todo, pues no me importaba o no me molestaba ver según qué cosas" (Pedro). Sin embargo los que se enfrentaron por primera vez a determinadas actividades relatan unos primeros momentos de incertidumbre e incluso angustia que dan paso al descubrimiento de unas capacidades que no pensaban que tenían. De hecho, se enfrentan a una actividad que tanto ellos como buena parte de su entorno tenían en gran desconsideración. "Nunca pensé que podría hacer lo que hago", nos dice Carles. Y es también el caso de Joan, que tampoco se veía como cuidador, pero superados los miedos iniciales y sintiéndose valorado por las que personas con las que trabaja, encuentra un sentido al cambio que se vio obligado a realizar:

"Yo decía: es que no me veo (...) Te encuentras con casos muy difíciles y con gente que se lo hace todo encima (...) Todo eso lo he superado, vi que lo podía hacer, que no me hacía, yo qué sé, dile asco... Por parte de la familia, de todos los pacientes que he hecho siempre me han valorado bien. Siempre, si he dejado un trabajo 'no lo dejes', al contrario, me han pasado a otros conocidos suyos porque han quedado contentos conmigo. $Y$ eso te hace pensar que... te ayuda a seguir".

Fernando, con un itinerario laboral similar, también explica con entusiasmo, cómo tras periodo inicial incierto por desconocimiento, supo transformar su recelo inicial hacia una ocupación que no había considerado nunca como tal en un trabajo que le permite sentirse bien. Un claro ejemplo de colono, según la tipología de Simpson (2004), con predisposición a la permanencia:

"Ahora no lo hago porque sí y ya, no lo hago por cualquier cosa, lo hago porque es mi profesión y porque es la profesión de mi vida. A mí alguien me pregunta si me cambiaria, no, yo no cambiaría. (...) Una vez me encontré a mi exjefe y me dice 'si volvieran los buenos tiempos de la obra, ¿volverías?’ La respuesta fue clara y sencilla, jamás (...) Yo entro aquí contento a trabajar y me voy contento (...)”.

Sin embargo, este no es el caso de Pedro, más buscador que colono, que cuando le preguntamos por qué trabaja como cuidador nos confiesa: “PPor qué? Básicamente por el dinero. Ya está. He estado aquí, he hecho esto, me lo estoy pasando bien, porque me lo estoy pasando muy bien. Ahora, el primer día que entré aquí dije: no me gustaría jubilarme de esto. Lo primero que dije. Pero bueno, todo de momento son agradecimientos y todo de 
momento $v a$ ". Esta última razón, la de la recompensa en forma de halagos por el trabajo realizado, es para muchos de ellos una de las que les ratifican su elección.

\section{EXPERIENCIAS DE LOS HOMBRES EN LOS TRABAJOS DE CUIDADOS EN SU CONDICIÓN MINORITARIA}

En un trabajo feminizado como es el de cuidados, los hombres traspasan las fronteras de género y rompen con la idea de masculinidad hegemónica cuando se identifican como cuidadores. Ellos piensan que son buenos profesionales independientemente del sexo: realizan las mismas tareas y se sienten bien aceptados por sus compañeras de trabajo aunque menos por parte de los receptores de cuidado (las mujeres son las que muestran mayores resistencias); obtienen el mismo salario, comparten la misma categoría profesional y mantienen su misma posición. Aun así, a pesar de manifestar su buen hacer y que pueden realizar las mismas tareas que las mujeres, hay distintos aspectos del trabajo, basados en la estructura de género, que marcan una diferencia en la atención que realizan. Así es como añaden dimensiones en el cuidado para distanciarse de aquello más feminizado o bien resaltan unas cualidades masculinizadas (la autoridad y la fuerza principalmente) que consideran necesarias para el cuidado.

Efectivamente, los hombres dicen realizar las mismas actividades de cuidado que las mujeres, definidas en función de las necesidades de la persona que han de atender, del ámbito competencial de la actividad laboral y del lugar donde trabajan. Algunos, para desmarcarse de la atención considerada más femenina, añaden a las tareas más asistenciales otras dimensiones como la educación, el ocio, la vigilancia y el dar conversación. Si bien su trabajo lo asimilan al de las mujeres, ellos dicen que aportan algo más que valoran positivamente y que los diferencia, redefiniendo la misma actividad.

"Es más ocio. Claro, harás duchas por si hay alguna urgencia, seguramente, pero es más ocio, es pasar el rato, un rato agradable, un rato divertido, que estés allí, vale, estás controlado, entre comillas, estas allí, pero estas pasando un rato divertido" (Fernando).

"Pues que necesita un control. Y el control primero es vigilancia, después ayuda y todo lo que representa: desde vestirlos a quitarles la mierda... Dar, no sé, cinco, diez, quince minutos... escucharlos" (Pedro).

Sólo establecen diferencias explícitas con las mujeres en tareas como la movilización de los receptores, las contenciones emocionales o los turnos de noche, que según el recurso, siempre realizan hombres. Estas funciones están directamente relacionadas con la condición física de la fuerza y la autoridad, cualidades masculinizadas y los identifica como hombres cuidadores, ya sea para dar un valor añadido confiriéndoles cierto poder por ser una minoría ya sea para reafirmar a la vez su masculinidad (Christensen y Knight, 2014), tal y como enfatiza Ernesto, "Yo pienso que la mujer (cuidadora) es más meticulosa, digo yo... Hace todo lo que hace un hombre, sólo que la fuerza la diferencia para hacer 
ciertos cambios". O bien, como señala Williams (1992), como una ventaja para ocupar estos trabajos siendo, por ello, más valorados:

“...como muchas personas se retrasaban y en las grúas, como yo estaba ahí en prácticas, pues acompañaba a la chica a levantarlo y llevarlo a la silla de ruedas para hacerle la ducha. Pero cuando ella está sola es muy complicado porque ella no lo puede hacer. Tendrá que pedir ayuda a las compañeras para poderlo hacer".

No obstante, la fuerza es relativizada como valor para el cuidado cuando existen otras técnicas que suplen esta cualidad humana. En la residencia asistida donde trabaja Arturo "si hay alguna movilidad compleja se ha de ayudar, no es dejarlo al hombre, porque han de ser dos personas cuando hay una movilidad muy compleja. Y, si no, están las famosas grúas. Yo las utilizo mucho".

A pesar de que los hombres no muestran preferencias en cuidar hombres o mujeres, existen barreras en relación al género marcadas principalmente por las personas receptoras de cuidado. Las mujeres suelen mostrar más resistencias a la realización de higiene íntima por parte de un cuidador mientras que son mejor aceptados por parte de los hombres.

"Hay algunas que les miras la cara y el reflejo de la cara te dice: no, tú no. No te lo dicen directamente, tú ya ves que te dicen que no. Y llamas a otra compañera y te contesta ¿cómo que no quiere?, ¿te ha dicho que no quiere? Y digo, no hace falta que me lo diga. Y entonces ellas hablan. Y no es porque no lo quiera hacer, es que yo pienso que se ha de respetar la intimidad. Sea hombre o mujer" (Arturo).

Cabe señalar que en el ámbito hospitalario y en los recursos para atender la discapacidad o la enfermedad mental no aparecen tantas resistencias, ya que la entrada del hombre a esta actividad laboral lleva más tiempo produciéndose. Sea donde fuere, para romper estas barreras muchas veces utilizan argumentos como "soy como un enfermero" o "soy como un médico", que resultan ser estrategias para negociar su masculinidad o incluso para renombrar su categoría laboral asimilándose a una categoría superior y menos feminizada (Simpson, 2004).

Los cuidadores entrevistados no nos han manifestado que ejerciendo como tales vean cuestionada su identidad masculina, sin embargo la redefinen mediante estrategias en la especificación y delimitación del cuidado y en las tareas que les son propias como cuidadores o resaltando aspectos de su masculinidad. Ellos incluyen tanto la parte psicológica y emocional como la parte física en el cuidado. El afecto (feminizado) no es un aspecto del cuidado que manifiesten de forma explícita, pero sí que todos ellos dicen implicarse emocionalmente. En este sentido se requiere de unas cualidades feminizadas (tener paciencia, empatía) que dicen adquirir mediante la formación, y unas cualidades masculinas (autoridad, fuerza, comunicación) que no se aprenden. En este sentido, el 
perfil del cuidador no solo demanda de las tradicionales cualidades femeninas, sino también de aquellas tradicionalmente asociadas a los hombres, lo que supone una redefinición del perfil laboral que legitima su presencia en el sector. Lo expresa muy bien Fernando:

“Ana también tiene un carácter fuerte, también. (...) Ella muchas veces es más autoritaria que yo. Yo lo veo, hostia! porque a ver, imagínate, como eres el hombre, y tienes la imagen del hombre, pues el hombre tiene que ser el autoritario, ¿no?”.

Todos ellos, sin excepción, diferencian las tareas de cuidado de las tareas de limpieza, como si se tratara de dimensiones paralelas. Hay que tener en cuenta que en algunos servicios (centros residenciales, servicios de ayuda a domicilio y centros sociosanitarios) estas dos actividades están delimitadas y especificadas como ocupaciones diferenciadas, pero no tanto en los domicilios particulares. Si tenemos en cuenta que el cuidado también incluye la atención y la limpieza del entorno, esto nos muestra la dificultad en delimitar las fronteras del trabajo reproductivo en un entorno institucional o productivo y, por lo tanto, la complejidad en la delimitación del cuidado cuando es remunerado. Ellos dicen que la limpieza no forma parte de su trabajo de una manera más contundente que sus colegas mujeres. El género en este sentido marca unos límites del cuidado, no sólo por el tipo de servicio y la profesión sino por el hecho de ser hombre o mujer. Así de claro lo argumenta Ernesto que trabaja en un servicio de ayuda a domicilio:

"En este caso yo no hago limpieza. En la misma empresa tenemos servicio de limpieza. Y hay usuarios que según las necesidades, esto lo valora la asistenta social, creo, necesitan esta persona" (Ernesto).

Joan incluso rechazó un trabajo en un domicilio particular porque se le pedía también realizar tareas de limpieza del domicilio:

"Me dijo 'tienes que hacer las camas a conciencia porque tengo gatos'. Después me lleva a la habitación del hijo, que también la tenía que arreglar y también el baño: 'en el baño, si lo mojas siempre lo secas y lo limpias'. Me lleva al otro baño y me dice que un día a la semana se tienen que hacer los espejos. Bueno, basta! Soy auxiliar geriátrico no personal de limpieza. No cogí este trabajo. Ahora pongo condiciones antes: si te interesa, sí, pero si no..." (Joan).

Ellos argumentan que proporcionan tan buena atención como cualquier mujer. Aun así, algunos sí creen que el género marca una diferencia en el tipo de atención. Jaume y Pedro, por ejemplo resaltan su masculinidad considerando que con los hombres se sienten más a gusto, pues el trato es diferente, más fácil y les pueden dar una mayor atención. Si el receptor es mujer explican que la relación es más compleja sobre todo, como hemos dicho anteriormente, en lo relacionado con la gestión de la intimidad corporal; además los temas de conversación son distintos, han de tener más cuidado, tratarlas con más deferencia, etc. 
"Y las mujeres sois de otra manera y se os ha de hablar de otra manera y de otros temas. De fútbol no le vas a hablar a una mujer, te van a mandar a paseo (...). Y a una mujer que le preguntas por sus hijos, te cuenta vida y milagros y ya te la has ganado" (Jaume).

"Con los tíos, el vocabulario normal, hostia, no seas 'malparit'! por ejemplo, no? Y hostia... y ya está, con una mujer como le digas que es una 'malparida', es que ya nos encendemos $y$ ya estamos quince días que ni nos hablamos. Entonces no es el mismo trato" (Pedro).

Debido a su presencia minoritaria en el sector de cuidados, cuando afirman que tienen la misma capacidad que las mujeres para el cuidado, expresan de distintas formas el efecto comodidad (Simpson, 2004) y en ningún caso ven cuestionada su masculinidad. Muchas veces reconocen la superioridad de las mujeres en los cuidados, tal como lo expresa Roberto: "Las heroinas, por llamarlo de alguna manera, siempre han sido las mujeres, las que han llevado este tema". Y algunos, como Carles, consideran que tienen que aprender mucho de la experiencia de las mujeres:

"Yo creo que, al final, con la experiencia, puedes llegar a tener la misma. No digo que pueda superar a una mujer ni mucho menos. Pero creo que podemos estar a la misma altura. Totalmente. Yo me estoy relacionando con auxiliares, que tienen muchos años de experiencia. Evidentemente saben mucho más que yo. Y cuando veo cosas digo: Me lo apunto".

Otros, como Roberto, se asimilan a ellas, porque les gusta trabajar con mujeres "Me tienen superbién cuidado, me miman mucho, hacemos bromas... a veces les digo: soy una más: en vez de uno digo una. Eso de separar, porque tú eres un chico, es una tontería...". Expresa muy bien la aceptación de los hombres en entornos de trabajo feminizados, que es otro de los elementos que forman parte de la "escalera de cristal" y que favorecen su inserción.

\section{CONCLUSIONES}

En el presente artículo nos hemos acercador a la experiencia de los hombres empleados en trabajos de cuidados, es decir que desempeñan estas labores de manera remunerada tanto en servicios públicos como privados, para valorar cómo impacta su presencia en estas ocupaciones, muy feminizadas, y cómo este trabajo les impacta a ellos mismos.

Respecto a las circunstancias de acceso a los empleos de cuidado hemos comprobado cómo una gran mayoría de los casos estudiados comparten itinerarios laborales similares. Hombres que han desarrollado una dilatada carrera laboral en ocupaciones con fuerte presencia masculina, que se han visto obligados a reconducir sus itinerarios como consecuencia la última crisis económica, iniciada en 2008, y buscar una salida laboral que les ha encaminado al descubrimiento y ejercicio de este tipo de trabajos. Algunos han descubierto que se sienten bien ejerciendo esta profesión y deciden quedarse; han dirigido o han encauzado activamente sus itinerarios laborales hacia trabajos relacionados con el 
cuidado y la ayuda a personas dependientes. Algunos de ellos, aluden a su experiencia (premonitoria) de cuidado de personas dependientes en el propio domicilio (padres, esposa...) como el aval necesario para desempeñar adecuadamente estas actividades. Unas actividades que forman parte de los propios componentes de la cultura del trabajo de cuidados en la que, ya sea desempeñado por hombres o por mujeres, se traspasan las fronteras (muy difusas) entre lo doméstico y lo público, de manera que el ámbito doméstico se proyecta en el laboral diluyendo la dicotomía entre trabajo productivo y reproductivo. Con formaciones más o menos especializadas, el trabajo de cuidados ha representado para los hombres una oportunidad ya que se trata de un sector laboral con mucho movimiento y donde la exigua presencia de hombres les vuelve atractivos por las posibilidades de aportar nuevos puntos de vista, maneras de hacer, dinámicas relacionales, etc.

Respecto a cómo experimentan su actividad en relación al género, hemos visto que en general los hombres cuidadores no se sienten diferentes en un entorno de trabajo mayoritariamente femenino. Aunque destacan otros factores que sí marcan diferencias en la realización del trabajo de cuidados como el perfil del receptor (si es una persona mayor, una persona discapacitada...), el sexo de la persona receptora (fundamentalmente por las resistencias de las mujeres receptoras), la rama profesional (sanitaria, social), el tipo de recurso (ámbito sanitario o social) y el espacio donde se lleve a cabo el cuidado (institución o domicilio). No obstante se hacen evidentes aquellos aspectos que dan continuidad a la estructura de género: 1) la cualidad física de la fuerza asociada al hombre, que muchas veces justifica su presencia en este sector feminizado; 2) las resistencias de las mujeres receptoras de cuidado a que las cuide un hombre, y 3) una desvinculación de la forma de cuidar considerada más femenina, añadiendo a las tareas asistenciales otras dimensiones complementarias. El trabajo de cuidados no cuestiona su identidad masculina, pero para ello en el lugar de trabajo se utilizan estrategias de distanciamiento emocional, como el hecho de incidir en la importancia técnica de determinadas actividades, reafirmarse ante los receptores asimilándose a médicos o enfermeros, lo que a su vez marca una distancia entre ellos y los receptores de cuidados, o desvinculando las actividades propias del trabajo doméstico de las del trabajo de cuidados.

Nos preguntábamos al inicio si para los hombres que ejercen trabajos de cuidados funciona la "escalera de cristal", como se ha mostrado en la enfermería, magisterio o trabajo social. Una de las diferencias importantes respecto a estas ocupaciones, tal como hemos señalado, es que los trabajos de cuidados se configuran como empleos mucho más tarde y en pleno predominio de la lógica neoliberal, están teniendo dificultades para convertirse en profesiones porque carecen de cualificación, credenciales y normativas claras, la posibilidad de carrera profesional es muy limitada y se restringe a la gestión y organización de los cuidados, y, finalmente, el peso de la lógica doméstica y de la 
naturalización impregna el sector. Todos estos factores contribuyen a que sea un sector más generizado y etnicizado que otros sectores ocupacionales, caracterizado por una gran precariedad laboral. Sin embargo para los hombres el trabajo de cuidados se ha configurado como una oportunidad para poder trabajar y el hecho de ser hombres les favorece: discriminación positiva en el acceso, trato complaciente por parte del entorno y facilidades para elegir servicio. Sin embargo, a diferencia de otros sectores, no hay posibilidades de ascenso, y la precariedad la sufren tanto los hombres como las mujeres. La respuesta, entonces, a la pregunta es sí, la "escalera de cristal" funciona para los hombres que se introducen en los trabajos de cuidados, pero de una forma más limitada, con menos recorrido que en otras ocupaciones, porque el trabajo de cuidados es en sí mismo un "techo de un cristal" más bien opaco tanto para hombres como para mujeres. 


\section{BIBLIOGRAFÍA CITADA}

Calasanti, Toni y Bowen, Mary Elizabeth (2006) "Spousal caregiving and crossing gender boundaries: Maintaining gender identities”. Journal of Aging Studies 20, pp. 253-263.

Carrasco, Cristina; Borderías, Cristina y Torns, Teresa (eds.) (2011) El trabajo de cuidados. Historia, teoría y políticas. Madrid: La Catarata.

Catanzaro, Raimondo y Colombo, Asher (eds.) (2009) Badanti \& Co. Il lavoro domestico straniero in Italia. Bologna: Il Mulino.

Christensen, Martin y Knight, Jessica (2014) “'Nursing is no place for men'. A thematic analysis of male nursing students experiences of undergraduate nursing education". Journal of Nursing Education and Practice 12, pp. 95-104.

Comas d'Argemir, Dolors(1995) Trabajo, género y cultura. Laconstrucción de desigualdades entre hombres y mujeres. Barcelona: Icaria.

Comas d’Argemir, Dolors (2015) "Los cuidados de larga duración y el cuarto pilar del Estado del bienestar". Revista de Antropología Social 24, pp. 173-196.

Connell, Robert y Messerschmidt, James (2005) "Hegemonic masculinity: rethinking the concept". Gender \& Society 19, pp. 829-859.

Cottingham, Marci D. (2014) "Recruiting men, constructing manhood: How health care organizations mobilize masculinities as nursing recruitment strategy". Gender \& Society 28, pp. 133-156.

Deusdad, Blanca, Comas d'Argemir, Dolors y Ddziegielewski, Sophie (2016) "Restructuring Long-Term Care in Spain: The Impact of The Economic Crisis on Public Policies and Social Work Practice", Journal of Social Service Research 42(2), pp. 246-262.

Ducharme, Francine; Lévesque, Louise, Lachance, Lise et al (2006) "Older husbands as caregivers of their wives: A descriptive study of the context and relational aspects of care". International Journal of Nursing Studies 43, pp. 567-579.

Durán, Ma Ángeles (2014) "Mujeres y hombres ante la situación de dependencia". Seminario Políticas públicas de atención a personas mayores dependientes: Hacia un sistema integral de cuidados. Madrid: Instituto de Mayores y Servicios Sociales 
Esquivel, Valeria (2011) La economía del cuidado en América Latina: poniendo a los cuidados en el centro de la agenda. PNUD.

Evans, Joan (1997) "Men in nursing: issues of gender segregation and hidden advantage". Journal of Advanced Nursing 26(2), pp. 226-231.

Fantova, Fernando (2013) "Políticas públicas y profesionalización de cuidados". Jornada Los servicios de cuidados de larga duración: retos de futuro ante el bienestar cotidiano. Barcelona: Universitat Autònoma de Barcelona.

Fraser, Nancy J. (2011) "Feminism, Capitalism and the Cunning of History". New Left Review 56, pp. 97-117.

Haile, Aster G. y Siegman. Karin A. (2014) "Masculinity at work. Intersectionality and identity constructions of migrant domestic workers in the Netherlands". En ThanhDam Truong et al. (eds.) Migration, Gender and Social Justice: Perspectives on Human Insecurity. Springer Bewrlin Heidelberg, pp. 105-119.

Hanlon, Niall (2012) Masculinities, care and equality: identity and nurture in men's lives. London: Palgrave and MacMillan.

Informe ACRA (2013) Informe econòmic i social ACRA del sector d'atenció a les persones amb dependència a Catalunya. Serveis residencials i d'atenció diürna. En línea: https://www. inforesidencias.com/resources/public/biblioteca/documentos/sociosanitario/201306informe-acra-esade.pdf

Lethbridge, Jane (2013) Care home versus home care? Which direction for care services in Europe? Eligibility for European Works Councils. Project Report. European Federation of Public Services Unions (EPSU), Brussels, Belgium.

Milne, Alisoun y Hatzidimitriadou, Eleni (2003) “'Isn't he wonderful?' Exploring the contribution and conceptualization of older husbands as carers". Ageing International 28(4), pp. 389-407.

Ministerio de Trabajo y Asuntos Sociales (2004) Libro Blanco de Atención a las Personas en Situación de Dependencia en España. En línea: http://www.dependencia.imserso.es/ InterPresent1/groups/imserso/documents/binario/libroblanco.pdf

Marbán, Vicente (2011) "Los actores sociales en el sistema de dependencia español". Cuaderno de Relaciones Laborales 29 (1), pp. 69-91. 
Martínez-Buján, Raquel (2014) "Los modelos territoriales de organización social del cuidado a personas mayores en los hogares". Revista Española de Investigaciones Sociológicas 145, pp. 99-126.

Moreno, Isidoro (1997) "Trabajo, ideologías sobre el trabajo y culturas del trabajo". Revista Andaluza de Relaciones Laborales 3, pp. 9-28.

Otaegui, Amaia (2007) "Presentación”. En Ricardo Escudero (coord.) La calidad del empleo del nuevo sistema para la autonomía personal y la atención a la dependencia. València: Germania, pp. 21-33.

Palenzuela, Pablo (1995) "Las culturas del trabajo: una aproximación antropológica". Sociología del Trabajo 24, pp. 3-28.

Pérez, Amaia (2006) "Amenaza Tormenta: La crisis de los cuidados y la reorganización del sistema económico". Revista de Economía Crítica 5, pp.7-37.

Peterson, Elin (2015) "Framing caregiving work for older people in spanish public policy: gender, power and social justice”. Revista Española de Ciencia Política 39, pp. 221-237.

Pickard, Linda (2012) "Substitution between formal and informal care: a natural experiment in social policy in Britain between 1985 and 2000". Ageing and Society 32, pp. 1147-1175.

Quartararo, Cristina y Falcinelli, Daniela (2013) "Not only men but also migrants in non-traditional occupations". International Review of Sociology 23(2), pp. 363-378.

Razavi, Shahra, y Staab, Silke (2010) "Mucho trabajo y poco salario. Perspectiva internacional de los trabajadores del cuidado". Revista Internacional del Trabajo 129(4), pp. $449-467$.

Recio, Carolina (2010) "Familismo, asitencialismo y precariedad. La configuración del empleo en el sector de atención a las personas en España”. Alternativas 17, pp. 19-44.

Recio, Carolina; Moreno-Colom, Sara; Borràs, Vicent y Torns, Teresa (2015) "La profesionalización del sector de los cuidados". Zerbituan 60, pp. 179-193.

Ribero, Oscar (2005) "Quando o cuidador e um homem". En María Constança Paúl y António Manuel Fonseca (eds.) Envelhecer em Portugal. Lisboa: Climepsi. FALTAN PÁGINAS 
Rodríguez, Gregorio (2007) "La protección social de la dependencia en España. Un modelo sui generis de desarrollo de los derechos sociales". Política y Sociedad 44 (2), pp. 69-85.

Rodríguez, Gregorio (2011) "Políticas sociales de atención a la dependencia en los Regímenes de Bienestar de la Unión Europea”. Cuaderno de Relaciones Laborales 29 (1), pp. 13-42.

Rogero-García, Jesús y Ahmed-Mohamed, Karim (2014). "What is the best care for community-dwelling dependent adults? Sources of care and perception of unmet needs in Spain". Revista Internacional de Sociología 72(2), pp. 403-4

Scrinzi, Francesca (2005) «Les hommes de ménage, ou comment aborder la féminisation des migrations en interviewant des hommes». Migrations et sociétés 17, pp. 99-100.

Shen-Miller, David y Smiler, Andrew (2005) "Men in female - dominated vocations. A rationale for academic study and introduction to the special issue". Sex Roles 72, pp. 269276.

Simpson, Ruth (2004) "Masculinity at Work: the Experiences of Men in Female Dominated Occupations". Employement and Society 18(2), pp. 349-368.

Smith, Ryan A. (2012) "Money, Benefits and Power: a Test of the Glass Ceiling and Glass Escalator Hypotheses”. Annals, AAPSS 653, pp. 149-172.

Tobío, Constanza; Agulló, María Silveria; Gómez, María Victoria y Martín, María Teresa (2010) El cuidado de las personas. Un reto para el siglo XXI. Barcelona: Fundació La Caixa.

Torns, Teresa, Borràs, Vicent, Moreno, Sara y Recio, Carolina (2014) Nuevas profesiones para la organización social del cuidado cotidiano. Memoria final de investigación para la Ministerio de Economía y competitividad.

Vilà, Antoni (2013) "Evolución y reforma del sistema para la autonomía y atención a la dependencia”. Actas de la Dependencia. Fundación Caser 8, pp. 49-69.

West, Candace y Zimmerman, Don (1987) “Doing Gender". Gender \& Society 1(2), pp. 125-151.

Williams, Christine (1992) "The Glass Escalator: Hidden Advantages for Men in the "Female" Professions". Social Problems 39(3), pp. 253-267. 
Williams, Christine (2013) "The Glass Escalator Revisited: Gender Inequality in Neoliberal Times, SWS Feminist Lecturer". Gender \& Society 27(5), pp. 609-629.

Williams, Christine (2015) "Crossing Over: Interdisciplinary Research on 'Men who do Women's Work"'. Sex Roles 72, pp. 390-395.

Woodhams, Carol, Lupton, Ben y Cowling, Marc (2015) "The Presence of Ethnic Minority and Disabled Men in Feminised Work: Intersectionality, Vertical Segregation and the Glass Escalator". Sex Roles 72, pp. 277-293. 